\title{
Discontinuous behavior of the Pauli potential in density functional theory as a function of the electron number
}

\author{
Eli Kraisler $\oplus^{1, *}$ and Axel Schild $\oplus^{2, \dagger}$ \\ ${ }^{1}$ Fritz, Haber Center for Molecular Dynamics and Institute of Chemistry, The Hebrew University of Jerusalem, 9091401 Jerusalem, Israel \\ ${ }^{2}$ Laboratorium für Physikalische Chemie, ETH Zürich, 8093 Zürich, Switzerland
}

(Received 26 September 2019; accepted 3 December 2019; published 14 February 2020)

\begin{abstract}
The Pauli potential is an essential quantity in orbital-free density functional theory (DFT) and in the exact electron factorization method for many-electron systems. Knowledge of the Pauli potential allows the description of a system relying on the density alone, without the need to calculate the orbitals. In this work, we explore the behavior of the exact Pauli potential in finite systems as a function of the number of electrons, employing the ensemble approach in DFT. Assuming the system is in contact with an electron reservoir, we allow the number of electrons to vary continuously and to obtain fractional as well as integer values. We derive an expression for the Pauli potential for a spin-polarized system with a fractional number of electrons, and we find that when the electron number surpasses an integer, the Pauli potential jumps by a spatially uniform constant, similarly to the Kohn-Sham potential. The magnitude of the jump equals the Kohn-Sham gap. We illustrate our analytical findings by calculating the exact and approximate Pauli potentials for $\mathrm{Li}$ and $\mathrm{Na}$ atoms with fractional numbers of electrons.
\end{abstract}

DOI: 10.1103/PhysRevResearch.2.013159

\section{INTRODUCTION}

Density functional theory (DFT) [1] is the leading theoretical framework used to describe the electronic properties of matter [2-11]. It is successfully applied to a wide range of systems in chemistry, solid-state physics, and materials science. Usually, DFT is implemented within the Kohn-Sham (KS) approach [12], where a many-electron system of interacting electrons is described by the introduction of an auxiliary system of noninteracting electrons subject to one multiplicative potential, $v_{\mathrm{KS}}[n](\mathbf{r})$, termed the KS potential. This potential is chosen in such a way that the ground-state electron density in the auxiliary system reproduces the ground-state density of the original many-electron system. Given the KS potential, for the noninteracting electrons we solve the Schrödinger equation

$$
\left(-\frac{1}{2} \nabla^{2}+v_{\mathrm{KS}}[n](\mathbf{r})\right) \varphi_{i}(\mathbf{r})=\varepsilon_{i} \varphi_{i}(\mathbf{r})
$$

to obtain the orbitals, $\varphi_{i}(\mathbf{r})$, and construct from them the ground-state density, $n(\mathbf{r})[13,14]$.

There exists, however, an alternative to the KS approach. It is possible to derive a Schrödinger equation for the square root of the ground-state density, $n^{1 / 2}(\mathbf{r})$, directly [15-17]:

$$
\left(-\frac{1}{2} \nabla^{2}+v_{\mathrm{KS}}[n](\mathbf{r})+v_{\theta}[n](\mathbf{r})\right) n^{1 / 2}(\mathbf{r})=\mu n^{1 / 2}(\mathbf{r}) .
$$

\footnotetext{
*eli.kraisler@mail.huji.ac.il

†axel.schild@phys.chem.ethz.ch
}

Published by the American Physical Society under the terms of the Creative Commons Attribution 4.0 International license. Further distribution of this work must maintain attribution to the author(s) and the published article's title, journal citation, and DOI.
The potential in the above equation is a sum of the well-known KS potential, $v_{\mathrm{KS}}[n](\mathbf{r})$, and $v_{\theta}[n](\mathbf{r})$, termed [16] the Pauli potential. Generally speaking, the role of the Pauli potential is to account for the fact that we are describing a system of fermions (and not bosons), which are subject to Pauli's exclusion principle. The eigenvalue of Eq. (2), $\mu$, is the chemical potential of the system. Equation (2) is central in the orbital-free (OF) approach in DFT [18-24].

Originally, Eq. (2) was derived by analyzing the Euler equation for the KS system [15-17], and the subsequent introduction of the Pauli kinetic energy and the Pauli potential. However, the same result can be obtained also using the exact electron factorization (EEF) approach [25-30] to the manyelectron problem.

One advantage of the OF-DFT approach [Eq. (2)] over the KS approach [Eq. (1)] is evident: one has to solve the Schrödinger equation only once and find only one eigenfunction, the square root of the density, $n^{1 / 2}(\mathbf{r})$, instead of finding as many $\mathrm{KS}$ orbitals $\varphi_{i}(\mathbf{r})$ as the number of electrons in the system (at least). However, this advantage comes with a price: for Eq. (2) to be of practical use in electronic structure calculations, an expression for the Pauli potential, $v_{\theta}[n](\mathbf{r})$, in terms of the density, $n(\mathbf{r})$, has to be known-exactly or approximately. This is in addition to the standing task of expressing the KS potential, $v_{\mathrm{KS}}[n](\mathbf{r})$, in terms of the densitya task that emerges also within the KS approach to DFT.

A general expression for $v_{\theta}[n](\mathbf{r})$ in terms of the density is not known, but several approximations to it have been suggested (see [17,21,24,31-36] and references therein) and applied to selected atoms [22,37,38] and molecules [21,23,31]. Furthermore, there exists an exact expression for $v_{\theta}[n](\mathbf{r})$ in terms of the KS orbitals and energy levels [17]. Although it cannot be directly applied in Eq. (2), it can serve as a starting 
point for exploring the properties of the Pauli potential and suggesting approximations for it.

Several analytical properties of the Pauli potential have been discovered during the years. The positivity of the potential [15,31], its coordinate scaling [17], the cusp in the vicinity of the nucleus [38], conditions on the gradient expansion [17], as well as properties for spherically symmetric systems $[39,40]$ and particularly for two- and three-level atoms and ions [41] have been discussed in the literature.

In this article, we focus on the behavior of the Pauli potential as a function of the number of electrons in the system, $N$. The system is in contact with an electron reservoir, which allows $N$ to vary continuously and obtain fractional as well as integer values. In the KS approach, we know that $v_{\mathrm{KS}}[n](\mathbf{r})$ experiences a discontinuous jump as $N$ crosses an integer [42-50]. What happens to the Pauli potential, $v_{\theta}[n](\mathbf{r})$, in this regard? This is the subject of the present work.

The article is organized as follows. In Sec. II we consider an interacting system and a noninteracting KS system with a varying number of electrons, we describe them within the ensemble approach in DFT [51], and we present the main quantities to be used in the following sections. Next, in Sec. III we obtain a generalized form for the Pauli potential, $v_{\theta, \sigma}[n](\mathbf{r})$, for the case of a spin-resolved system with fractional $N$. In Sec. IV we explicitly take the limit of integer number of electrons, from below and from above, and we analyze the behavior of $v_{\theta, \sigma}[n](\mathbf{r})$ as $N$ surpasses an integer. After providing the numerical details in Sec. V, we illustrate our analytical findings in Sec. VI with numerical results for the $\mathrm{Li}$ and $\mathrm{Na}$ atoms. Finally, in Sec. VII we summarize and discuss our results.

\section{ENSEMBLE STATES IN MANY-ELECTRON SYSTEMS}

Strictly speaking, there is no such thing as a closed physical system with a fractional number of electrons, because electrons do not fracture in any system of our interest. The concept of a fractional number of electrons may, however, be introduced as a time average of the number of electrons in an open system, namely in a system that is free to exchange electrons with its surroundings (Ref. [52], Sec. 14). The ground state of such a system can no longer be described by a pure quantum-mechanical state. Instead, a statistical mixture, or ensemble, of pure (integer-electron) states has to be considered [51].

In the following, we analyze a system with $N=N_{0}+\alpha$ electrons, where $N_{0}$ is an integer number, corresponding to the number of electrons in an electrically neutral system, and $0 \leqslant$ $\alpha \leqslant 1$. Relying on the convexity conjecture for many-electron systems [3,51,53-55], the ensemble ground state consists only of the pure ground states for $N_{0}$ and $N_{0}+1$ electrons, $\left|\Psi_{N_{0}}\right\rangle$ and $\left|\Psi_{N_{0}+1}\right\rangle$ :

$$
\hat{\Lambda}=(1-\alpha)\left|\Psi_{N_{0}}\right\rangle\left\langle\Psi_{N_{0}}|+\alpha| \Psi_{N_{0}+1}\right\rangle\left\langle\Psi_{N_{0}+1}\right|,
$$

with the statistical weights of $(1-\alpha)$ and $\alpha$, respectively $[3,51,53,56,57]$. As a direct consequence of Eq. (3), the expectation value of any operator $\hat{O}$ in the ensemble state is given by $O=\operatorname{Tr}\{\hat{\Lambda} \hat{O}\}=(1-\alpha)\left\langle\Psi_{N_{0}}|\hat{O}| \Psi_{N_{0}}\right\rangle+$
$\alpha\left\langle\Psi_{N_{0}+1}|\hat{O}| \Psi_{N_{0}+1}\right\rangle$ [51]. In particular, the density

$$
n(\mathbf{r} ; N)=(1-\alpha) n\left(\mathbf{r} ; N_{0}\right)+\alpha n\left(\mathbf{r} ; N_{0}+1\right)
$$

and the energy

$$
E(N)=(1-\alpha) E\left(N_{0}\right)+\alpha E\left(N_{0}+1\right)
$$

are piecewise-linear in $N$. Focusing on the energy, for any fractional $N, E(N)$ is linear, but it can change its slope when $N$ passes an integer. Consequently, the chemical potential, $\mu=\partial E / \partial N$, used in Eq. (2), is a stair-step function of $N$. For example, around $N=N_{0}$,

$$
\mu(N)=\left\{\begin{array}{l}
-I: N_{0}-1<N \leqslant N_{0}, \\
-A: N_{0}<N \leqslant N_{0}+1,
\end{array}\right.
$$

where $I=E\left(N_{0}-1\right)-E\left(N_{0}\right)$ is the ionization potential (IP) and $A=E\left(N_{0}\right)-E\left(N_{0}+1\right)$ is the electron affinity (EA). Clearly, the chemical potential is generally discontinuous at integer $N$.

Since in the following sections we derive the Pauli potential in terms of the KS system, we consider here also the KS system at fractional $N$. Not only is the ground state of the interacting system described by an ensemble, but also the KS system is in an ensemble state [58,59], which is given by

$$
\hat{\Lambda}_{\mathrm{KS}}^{(\alpha)}=(1-\alpha)\left|\Phi_{N_{0}}^{(\alpha)}\right\rangle\left\langle\Phi_{N_{0}}^{(\alpha)}|+\alpha| \Phi_{N_{0}+1}^{(\alpha)}\right\rangle\left\langle\Phi_{N_{0}+1}^{(\alpha)}\right|,
$$

where $\left|\Phi_{N_{0}}^{(\alpha)}\right\rangle$ and $\left|\Phi_{N_{0}+1}^{(\alpha)}\right\rangle$ are pure KS states, with $N_{0}$ and $N_{0}+1$ particles, respectively $[13,60]$. Both of the aforementioned pure states are given by Slater determinants constructed out of single-electron orbitals $\left\{\varphi_{i}^{(\alpha)}(\mathbf{r})\right\}$, which correspond to the same $\mathrm{KS}$ potential, $v_{\mathrm{KS}}^{(\alpha)}(\mathbf{r})$. The dependence of the KS potential, and as a result the orbitals and the Slater determinants, on the number of electrons, i.e., on $\alpha$, is emphasized by the superscript $(\alpha)$. This is to say that when varying $\alpha,\left|\Phi_{N_{0}}^{(\alpha)}\right\rangle$ and $\left|\Phi_{N_{0}+1}^{(\alpha)}\right\rangle$ change due to the change in $v_{\mathrm{KS}}^{(\alpha)}(\mathbf{r})$, unlike $\left|\Psi_{N_{0}}\right\rangle$ and $\left|\Psi_{N_{0}+1}\right\rangle$, which are $\alpha$-independent. As a direct consequence of Eq. (7), the expectation value of any operator $\hat{O}$ in the ensemble state is

$$
O=(1-\alpha)\left\langle\Phi_{N_{0}}^{(\alpha)}|\hat{O}| \Phi_{N_{0}}^{(\alpha)}\right\rangle+\alpha\left\langle\Phi_{N_{0}+1}^{(\alpha)}|\hat{O}| \Phi_{N_{0}+1}^{(\alpha)}\right\rangle .
$$

From the above equation, after some algebraic manipulations, one can obtain the ensemble density as $n(\mathbf{r})=$ $\sum_{i=1}^{\infty} f_{i}\left|\varphi_{i}^{(\alpha)}(\mathbf{r})\right|^{2}$, where the occupation numbers $f_{i}$ are defined as

$$
f_{i}=\left\{\begin{array}{l}
1: i \leqslant N_{0}, \\
\alpha: i=N_{0}+1, \\
0: i>N_{0}+1,
\end{array}\right.
$$

meaning that the first $N_{0} \mathrm{KS}$ energy levels are fully occupied, the $\left(N_{0}+1\right)$ th level is occupied by a fraction of an electron, $\alpha$, and all the higher-lying levels are vacant. Similarly, the KS kinetic energy of the ensemble state can be expressed as $T_{\mathrm{KS}}[n]=\sum_{i=1}^{\infty} f_{i}\left\langle\varphi_{i}^{(\alpha)}\left|-\frac{1}{2} \nabla^{2}\right| \varphi_{i}^{(\alpha)}\right\rangle$. We stress that the fractional occupation numbers $f_{i}$, as given by Eq. (9), appear in the expressions for $n(\mathbf{r})$ and $T_{\mathrm{KS}}[n]$ (and other quantities below) as a direct and inevitable consequence of Eq. (8), which in turn directly follows from the definition of the ensemble state in the Kohn-Sham system, Eq. (7). Introduction of the occupation numbers $f_{i}$ is neither our choice nor an 
empirical ansatz. For the case of an integer $N$, e.g., for $N=$ $N_{0}$, the equations for the density and the kinetic energy reduce to their familiar forms, $n(\mathbf{r})=\sum_{i=1}^{N_{0}}\left|\varphi_{i}^{(0)}(\mathbf{r})\right|^{2}$ and $T_{\mathrm{KS}}[n]=$ $\sum_{i=1}^{N_{0}}\left\langle\varphi_{i}^{(0)}\left|-\frac{1}{2} \nabla^{2}\right| \varphi_{i}^{(0)}\right\rangle$.

Lastly, we note that from a combination of Janak's theorem [61] and Eq. (9), it follows that the highest-occupied (ho) KS energy level equals the chemical potential, $\mu(N)$ :

$$
\varepsilon_{\mathrm{ho}}(N)=\frac{\partial E(N)}{\partial f_{\mathrm{ho}}}=\frac{\partial E(N)}{\partial \alpha}=\frac{\partial E(N)}{\partial N}=\mu(N),
$$

and is also discontinuous at integer $N$ [see Eq. (6)]. Notably, the discontinuity in $\varepsilon_{\text {ho }}(N)$ comes from two sources (see Ref. [59] and references therein): First, as $N$ increases above an integer value, one starts to occupy a new level, which has remained vacant so far, namely the (former) lowest unoccupied level. For this reason, $\varepsilon_{\text {ho }}$ jumps by the difference $E_{g}^{\mathrm{KS}}=$ $\varepsilon_{\text {lu }}^{-}-\varepsilon_{\text {ho }}^{-}$, called the KS gap. The superscript (-) emphasizes the fact that the energy levels are taken for the $N_{0}$-electron system, in the limit $N \rightarrow N_{0}^{-}$. However, this jump is almost always insufficient to satisfy Eq. (6), because generally $\varepsilon_{\text {lu }}^{-} \neq$ $-A$, even for the exact $\mathrm{KS}$ potential. For this reason, the $\mathrm{KS}$ potential jumps by a spatially uniform constant as $N$ surpasses an integer (see Refs. [42,43,50,59] and references therein). This constant is usually denoted $\Delta$ and equals $-A+\varepsilon_{\text {lu }}^{-}$. It can also be expressed as $\Delta=E_{g}-E_{g}^{\mathrm{KS}}$, i.e., the difference between the fundamental gap, $E_{g}=I-A$ (namely, the difference between the IP and the EA), and the KS gap.

For simplicity, so far the formalism has been presented in the spin-unpolarized form. Generalization to the spinpolarized form, in the case in which only one spin channel is fractionally occupied (and therefore the ensemble is still comprised of two pure states), is straightforward. In particular, the total number of electrons is expressed as $N=N_{0 \uparrow}+$ $N_{0 \downarrow}+\alpha$, and the total density is $n(\mathbf{r})=n_{\uparrow}(\mathbf{r})+n_{\downarrow}(\mathbf{r})$, where $n_{\sigma}(\mathbf{r})=\sum_{i=1}^{\infty} f_{i \sigma}\left|\varphi_{i \sigma}^{(\alpha)}(\mathbf{r})\right|^{2}$ is the spin density in the channel $\sigma$ (the index $\sigma$ stands for $\uparrow$ or $\downarrow$ ). In the equation for the spin density, the occupation numbers equal

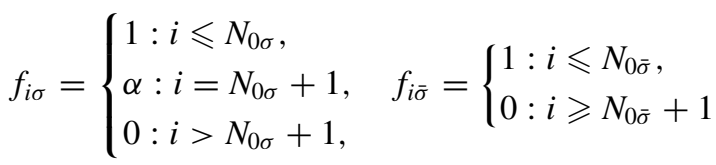

for the fractionally occupied and the integrally occupied channels, respectively. Here $\bar{\sigma}$ corresponds to the spin channel opposite to $\sigma$. Finally, the ho KS energy level in each spin channel equals $\varepsilon_{\mathrm{ho}, \sigma}(\alpha)=\partial E(\alpha) / \partial f_{\mathrm{ho}, \sigma}=\partial E(\alpha) / \partial N_{\sigma}$. The global ho level can be expressed as $\varepsilon_{\mathrm{ho}}=\max \left(\varepsilon_{\mathrm{ho}, \uparrow}, \varepsilon_{\mathrm{ho}, \downarrow}\right)$. It is the global ho level that equals the chemical potential $\mu(N)$. As $N$ surpasses an integer, the uniform jump $\Delta$ occurs in the $\mathrm{KS}$ potential of that channel to which the extra fraction of an electron is added.

\section{PAULI POTENTIAL FOR FRACTIONAL $N$}

Equipped with the mathematical tools required to address many-electron systems with ensembles, we proceed to derive the Pauli potential for the spin-polarized case with a fractional number of electrons. As already mentioned, we confine ourselves to the case in which only one spin channel is fractionally occupied. The result derived in this section is a generalization of the result for the exact Pauli potential derived in Ref. [17], in two respects: first, the derivation given below considers a spin-polarized many-electron system; second, a fractional number of electrons is explicitly examined.

We start with the Euler equation, in its spin-polarized form,

$$
\frac{\delta T_{\mathrm{KS}}\left[n_{\uparrow}, n_{\downarrow}\right]}{\delta n_{\sigma}(\mathbf{r})}+v_{\mathrm{KS}, \sigma}\left[n_{\uparrow}, n_{\downarrow}\right](\mathbf{r})=\varepsilon_{\mathrm{ho}, \sigma} .
$$

Then, the KS kinetic energy is expressed as

$T_{\mathrm{KS}}\left[n_{\uparrow}, n_{\downarrow}\right]=\sum_{\sigma} \int n_{\sigma}^{1 / 2}(\mathbf{r})\left(-\frac{1}{2} \nabla^{2}\right) n_{\sigma}^{1 / 2}(\mathbf{r}) d^{3} r+T_{\theta}\left[n_{\uparrow}, n_{\downarrow}\right]$,

where $T_{\theta}\left[n_{\uparrow}, n_{\downarrow}\right]$ is the Pauli kinetic energy; the latter energy is actually defined by the above expression. Next, the functional derivative of the KS kinetic energy is calculated, to yield

$$
\frac{\delta T_{\mathrm{KS}}\left[n_{\uparrow}, n_{\downarrow}\right]}{\delta n_{\sigma}(\mathbf{r})}=-\frac{1}{2} \frac{\nabla^{2}\left[n_{\sigma}^{1 / 2}(\mathbf{r})\right]}{n_{\sigma}^{1 / 2}(\mathbf{r})}+v_{\theta, \sigma}\left[n_{\uparrow}, n_{\downarrow}\right](\mathbf{r}),
$$

where $v_{\theta, \sigma}\left[n_{\uparrow}, n_{\downarrow}\right](\mathbf{r})=\delta T_{\theta, \sigma}\left[n_{\uparrow}, n_{\downarrow}\right] / \delta n_{\sigma}(\mathbf{r})$ is the spinpolarized Pauli potential. Introduction of Eq. (14) into Eq. (12) and rearrangement of terms yields the spin-polarized version of Eq. (2):

$$
\left(-\frac{1}{2} \nabla^{2}+v_{\mathrm{KS}, \sigma}(\mathbf{r})+v_{\theta, \sigma}(\mathbf{r})\right) n_{\sigma}^{1 / 2}(\mathbf{r})=\varepsilon_{\mathrm{ho}, \sigma} n_{\sigma}^{1 / 2}(\mathbf{r}) .
$$

We now derive an expression for the spin-polarized Pauli potential, $v_{\theta, \sigma}\left[n_{\uparrow}, n_{\downarrow}\right](\mathbf{r})$, for a system with fractional $N$. For the upcoming derivation, we introduce the quantities

$$
q_{i \sigma}(\mathbf{r})=\varphi_{i \sigma}(\mathbf{r}) / n_{\sigma}^{1 / 2}(\mathbf{r})
$$

These quantities possess the following properties:

(i) $\sum_{i=1}^{\infty} f_{i \sigma}\left|q_{i \sigma}(\mathbf{r})\right|^{2}=1$, as a direct consequence of the definition of the density $n_{\sigma}(\mathbf{r})$.

(ii) $\sum_{i=1}^{\infty} f_{i \sigma} \nabla\left|q_{i \sigma}(\mathbf{r})\right|^{2}=\sum_{i=1}^{\infty} f_{i \sigma} q_{i \sigma}^{*}(\mathbf{r}) \nabla q_{i \sigma}(\mathbf{r})+$ c.c. $=0$, as follows from applying the operator $\nabla$ to (i).

(iii) $\quad \sum_{i=1}^{\infty} f_{i \sigma} \nabla^{2}\left|q_{i \sigma}(\mathbf{r})\right|^{2}=\sum_{i=1}^{\infty} f_{i \sigma} q_{i \sigma}^{*}(\mathbf{r}) \nabla^{2} q_{i \sigma}(\mathbf{r})+$ $\nabla q_{i \sigma}^{*}(\mathbf{r}) \cdot \nabla q_{i \sigma}(\mathbf{r})+$ c.c. $=0$, as follows from applying $\nabla \cdot$ to (ii).

We start with the spin-polarized version of Eq. (1), express it in terms of $q_{i \sigma}(\mathbf{r})$ and $n_{\sigma}(\mathbf{r})$, rather than in terms of $\varphi_{i \sigma}(\mathbf{r})$ [62], then multiply it by $f_{i \sigma} q_{i \sigma}^{*}(\mathbf{r})$ from the left and sum over all $i$ to get

$$
\begin{aligned}
& \sum_{i=1}^{\infty} f_{i \sigma} q_{i \sigma}^{*}(\mathbf{r})\left(-\frac{1}{2} \nabla^{2}\right)\left[q_{i \sigma}(\mathbf{r}) n_{\sigma}^{1 / 2}(\mathbf{r})\right] \\
& \quad+v_{\mathrm{KS}, \sigma}(\mathbf{r}) n_{\sigma}^{1 / 2}(\mathbf{r}) \sum_{i=1}^{\infty} f_{i \sigma}\left|q_{i \sigma}(\mathbf{r})\right|^{2} \\
& =n_{\sigma}^{1 / 2}(\mathbf{r}) \sum_{i=1}^{\infty} f_{i \sigma} \varepsilon_{i \sigma}\left|q_{i \sigma}(\mathbf{r})\right|^{2} .
\end{aligned}
$$

The second term on the left-hand side (lhs) of Eq. (17) can be simplified using property (i) for $q_{i \sigma}(\mathbf{r})$. The right-hand side (rhs) of Eq. (17) can be rewritten as $n_{\sigma}^{1 / 2}(\mathbf{r})\left[\varepsilon_{\mathrm{ho}, \sigma}+\sum_{i=1}^{\infty} f_{i \sigma}\left(\varepsilon_{i \sigma}-\varepsilon_{\mathrm{ho}, \sigma}\right)\left|q_{i \sigma}(\mathbf{r})\right|^{2}\right]$, using again property (i). Furthermore, to simplify the first term on the lhs, 
we note that

$$
\nabla^{2}\left[q_{i \sigma}(\mathbf{r}) n_{\sigma}^{1 / 2}(\mathbf{r})\right]=q_{i \sigma}(\mathbf{r}) \nabla^{2}\left[n_{\sigma}^{1 / 2}(\mathbf{r})\right]+2 \nabla\left[n_{\sigma}^{1 / 2}(\mathbf{r})\right] \cdot \nabla q_{i \sigma}(\mathbf{r})+n_{\sigma}^{1 / 2}(\mathbf{r}) \nabla^{2} q_{i \sigma}(\mathbf{r}) .
$$

We substitute this form into Eq. (17) and use property (i) again to obtain

$$
\begin{aligned}
-\frac{1}{2} \nabla^{2}\left[n_{\sigma}^{1 / 2}(\mathbf{r})\right]+n_{\sigma}^{1 / 2}(\mathbf{r}) \cdot\{ & v_{\mathrm{KS}, \sigma}(\mathbf{r})+\sum_{i=1}^{\infty} f_{i \sigma}\left[q_{i \sigma}^{*}(\mathbf{r})\left(-\frac{1}{2} \nabla^{2}\right) q_{i \sigma}(\mathbf{r})-q_{i \sigma}^{*}(\mathbf{r}) \frac{\nabla\left[n_{\sigma}^{1 / 2}(\mathbf{r})\right] \cdot \nabla q_{i \sigma}(\mathbf{r})}{n_{\sigma}^{1 / 2}(\mathbf{r})}\right. \\
& \left.\left.+\left(\varepsilon_{\mathrm{ho}, \sigma}-\varepsilon_{i \sigma}\right)\left|q_{i \sigma}(\mathbf{r})\right|^{2}\right]\right\}=\varepsilon_{\mathrm{ho}, \sigma} n_{\sigma}^{1 / 2}(\mathbf{r}) .
\end{aligned}
$$

We now take the complex conjugate of Eq. (19), sum up the two equations, and divide by 2 . Using property (iii) for the first term in the square brackets and property (ii) for the second term there, we realize that Eq. (19) can be rewritten in the form of Eq. (15), where the Pauli potential equals

$$
\begin{aligned}
v_{\theta, \sigma}(\mathbf{r})= & \frac{1}{2} \sum_{i=1}^{\infty} f_{i \sigma} \nabla q_{i \sigma}^{*}(\mathbf{r}) \cdot \nabla q_{i \sigma}(\mathbf{r}) \\
& +\sum_{i=1}^{\infty} f_{i \sigma}\left(\varepsilon_{\mathrm{ho}, \sigma}-\varepsilon_{i \sigma}\right)\left|q_{i \sigma}(\mathbf{r})\right|^{2} .
\end{aligned}
$$

This concludes our derivation of the spin-polarized Pauli potential for a system with fractional number of electrons, which is the central result of this section. In the following sections we will separately address the two parts of the Pauli potential, $v_{\theta, \sigma}^{(1)}(\mathbf{r})=\frac{1}{2} \sum_{i=1}^{\infty} f_{i \sigma} \nabla q_{i \sigma}^{*}(\mathbf{r}) \cdot \nabla q_{i \sigma}(\mathbf{r})$ and $v_{\theta, \sigma}^{(2)}(\mathbf{r})=$ $\sum_{i=1}^{\infty} f_{i \sigma}\left(\varepsilon_{\mathrm{ho}, \sigma}-\varepsilon_{i \sigma}\right)\left|q_{i \sigma}(\mathbf{r})\right|^{2}$.

For completeness, we consider also the spin-polarized Pauli kinetic energy density per particle, $t_{\theta, \sigma}\left[n_{\sigma}\right](\mathbf{r})$, which serves in the integral representation $T_{\theta}[n]=$ $\sum_{\sigma} \int n_{\sigma}(\mathbf{r}) t_{\theta, \sigma}\left[n_{\sigma}\right](\mathbf{r}) d^{3} r$. Relying on the definition of $T_{\theta}\left[n_{\uparrow}, n_{\downarrow}\right]$ and performing the same manipulations as before, we find that

$$
t_{\theta, \sigma}\left[n_{\sigma}\right](\mathbf{r})=\frac{1}{2} \sum_{i=1}^{\infty} f_{i \sigma} \nabla q_{i \sigma}^{*}(\mathbf{r}) \cdot \nabla q_{i \sigma}(\mathbf{r}) .
$$

In the spin-unpolarized case with fractional occupations, the form of Eqs. (20) and (21) remains the same, but the occupation number $f_{i}$ equals 2 for the first $N_{0} / 2$ orbitals, $\alpha$ for the highest, fractionally occupied orbital, and zero otherwise. In the particular case of a spin-unpolarized system with integer $N$, Eqs. (20) and (21) reduce to their previously known forms [see Ref. [17], Eqs. (16) and (22), respectively], as expected.

\section{DISCONTINUOUS BEHAVIOR OF THE PAULI POTENTIAL AROUND AN INTEGER $N$}

In this section, we explicitly consider the case in which the number of electrons in the system reaches an integer from below, $N \rightarrow N_{0}^{-}$, and from above, $N \rightarrow N_{0}^{+}$, and we analyze the behavior of $v_{\theta, \sigma}(\mathbf{r})$ in these limits.

In the limit $N \rightarrow N_{0}^{-}$, the occupation numbers in both spin channels have the form

$$
f_{i \sigma}=\left\{\begin{array}{l}
1: i \leqslant N_{0 \sigma}, \\
0: i>N_{0 \sigma},
\end{array}\right.
$$

and the Pauli potential equals

$$
v_{\theta, \sigma}^{-}(\mathbf{r})=\sum_{i=1}^{N_{0}} \frac{1}{2} \nabla q_{i \sigma}^{*}(\mathbf{r}) \cdot \nabla q_{i \sigma}(\mathbf{r})+\left(\varepsilon_{\mathrm{ho}, \sigma}^{-}-\varepsilon_{i \sigma}^{-}\right)\left|q_{i \sigma}(\mathbf{r})\right|^{2} .
$$

All the quantities that comprise the Pauli potential $v_{\theta, \sigma}^{-}(\mathbf{r})$ are those corresponding to the limit $N \rightarrow N_{0}^{-}$, a fact that has been emphasized by a superscript $(-)$ for the eigenvalues (but not for the other quantities, for reasons that become apparent below).

As the number of electrons grows and infinitesimally surpasses an integer, the density and the KS orbitals change continuously, but the KS potential (of the channel to which the fraction of an electron is added) jumps by a spatially uniform constant $\Delta$, as mentioned in Sec. II. As a result, the KS eigenvalues of this spin channel change abruptly, by the same constant: $\varepsilon_{i \sigma}^{+}=\varepsilon_{i \sigma}^{-}+\Delta$, and in particular $\varepsilon_{\mathrm{ho}, \sigma}^{+}=$ $\varepsilon_{\mathrm{lu}, \sigma}^{-}+\Delta$ and therefore $\varepsilon_{\mathrm{ho}, \sigma}^{+}-\varepsilon_{i \sigma}^{+}=\varepsilon_{\mathrm{lu}, \sigma}^{-}-\varepsilon_{i \sigma}^{-}$. Then, in the $\operatorname{limit} N \rightarrow N_{0}^{+}$, the Pauli potential equals

$$
v_{\theta, \sigma}^{+}(\mathbf{r})=\sum_{i=1}^{N_{0}} \frac{1}{2} \nabla q_{i \sigma}^{*}(\mathbf{r}) \cdot \nabla q_{i \sigma}(\mathbf{r})+\left(\varepsilon_{\mathrm{lu}, \sigma}^{-}-\varepsilon_{i \sigma}^{-}\right)\left|q_{i \sigma}(\mathbf{r})\right|^{2} .
$$

Subtraction of Eq. (23) from Eq. (24) yields

$$
v_{\theta, \sigma}^{+}(\mathbf{r})-v_{\theta, \sigma}^{-}(\mathbf{r})=\sum_{i=1}^{N_{0}}\left(\varepsilon_{\mathrm{lu}, \sigma}^{-}-\varepsilon_{\mathrm{ho}, \sigma}^{-}\right)\left|q_{i \sigma}(\mathbf{r})\right|^{2}=E_{g}^{\mathrm{KS}} .
$$

Therefore, we conclude that the Pauli potential also jumps by a spatially uniform constant when $N$ surpasses an integer value. The magnitude of the constant is the $\mathrm{KS}$ gap, $E_{g}^{\mathrm{KS}}$. The jump in the Kohn-Sham-Pauli potential, i.e., the overall potential used in Eq. (2) for the square root of the density, equals the fundamental gap, $E_{g}$. Notably, whereas the height of the uniform jump experienced by the KS potential involves both quantities of the interacting system ( $I$ and $A$ ) and the $\mathrm{KS}$ system (ho and lu energies), the jump of the Pauli potential is expressed only in terms of KS orbital energies, and the KohnSham-Pauli gap_-only in terms of the interacting system.

\section{NUMERICAL DETAILS}

The numerical results presented in Sec. VI A are obtained by using the exact ground-state densities obtained with the full configuration-interaction (FCI) method for systems with integer $N$. In particular, we solved the many-electron problem for 
$\mathrm{Li}^{+}(\alpha=0)$ and $\mathrm{Li}(\alpha=1)$ using the FCI method in MOLPRO [63] with the Universal Gaussian Basis Set [64], employing the approach suggested in Ref. [65]. We then calculated the densities for fractional $N$ as in Eq. (4) with ORBKIT [66] on a logarithmic grid, linearly combining the exact densities of the cation and the neutral atom. Next, we employed the inversion procedure of Ref. [67] to obtain the KS potential $v_{\mathrm{KS}}^{(\alpha)}(\mathbf{r})$ and the resulting KS orbitals and eigenvalues (see also Ref. [50] and the supplemental material therein). Finally, we calculated the Pauli potential for each value of $\alpha$ in two ways: (i) by directly using Eq. (20), and (ii) by resolving Eq. (2) for the KS-Pauli potential, $v_{\mathrm{KS} \theta}(\mathbf{r})=\frac{1}{2} \frac{\nabla^{2} n^{1 / 2}(\mathbf{r})}{n^{1 / 2}(\mathbf{r})}+\varepsilon_{\text {ho }}$, and then subtracting the KS potential obtained by inversion. These two ways of calculating the Pauli potential are equivalent.

The results presented in Sec. VIB were obtained using the computer program ORCHID [68,69], version 4.0. The $\mathrm{Li}$ and $\mathrm{Na}$ atoms with fractional number of electrons were solved within the local spin-density approximation (LSDA) [70], in its standard implementation. The occupation numbers were set as in Eq. (11). The Pauli potential has been obtained again in two ways, similarly to what we described above for the FCI case, but in the spin-polarized version. In particular, to find the KS-Pauli potential in (ii), we address Eq. (15) instead of Eq. (2).

\section{COMPUTATIONAL RESULTS}

In this section we illustrate the findings of Secs. III and IV, particularly Eqs. (20) and (25), for selected atomic systems.

\section{A. Exact results}

Figure 1 presents the exact, spin-unpolarized Pauli potential for the $\mathrm{Li}$ atom, with a varying number of electrons, $N=2+\alpha$, with $\alpha=10^{-4}, 10^{-6}, 10^{-7}$, and $10^{-10}$. In panel (a) of Fig. 1, the total Pauli potential, $v_{\theta}(r)$, is depicted. Potentials obtained in ways (i) and (ii) (see Sec. V for details) are entirely on top of each other, as expected. The Pauli potential is positive everywhere [15,31], and for low values of $\alpha$ it has a characteristic form: it develops a plateau around the origin that drops to zero at far distances. As $\alpha \rightarrow 0^{+}$, the width of the plateau grows, ultimately reaching infinity. The plateau height approaches the value of $E_{g}^{\mathrm{KS}}$. This is in agreement with Eq. (25), because the Pauli potential for $\mathrm{Li}^{+}$is zero everywhere [71]. Qualitatively, the behavior of the Pauli potential is similar to the behavior of the KS potential at low $\alpha$ (compare to, e.g., [50]), albeit with a different plateau height.

To gain more understanding of the Pauli potential $v_{\theta}(r)$, we examine in panels (b) and (c) of Fig. 1 the two terms that form the Pauli potential: $v_{\theta}^{(1)}(r)$ [which also equals $t_{\theta}(r)$ ] and $v_{\theta}^{(2)}(r)$. We clearly see that the term that contributes to the creation of the plateau is $v_{\theta}^{(2)}(r)$, in agreement with Eq. (25). The term $v_{\theta}^{(1)}(r)$ contributes only in the region where the plateau drops to zero, effectively shifting the plateau edge to the right.

\section{B. The LSDA approximation}

In addition to the exact results presented above, we are interested in answering the question of how well approximate xc functionals capture the exact properties of the Pauli potential. It is well known that common xc approximations, such as the

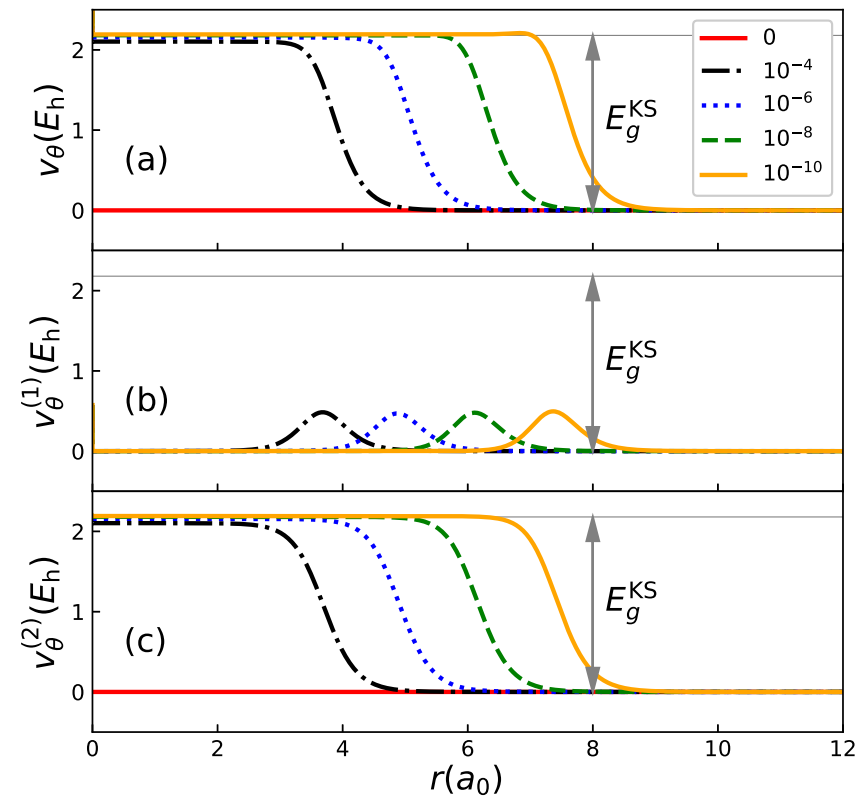

FIG. 1. (a) Exact total Pauli potential, $v_{\theta}(r)$, for the Li atom with $2+\alpha$ electrons, for various values of $\alpha$ (see the legend). Pauli potentials obtained in ways (i) and (ii) (see Sec. V) are indistinguishable. (b) The term $v_{\theta}^{(1)}(r)$ for the Li atom with $2+\alpha$ electrons, for various values of $\alpha$. (c) The term $v_{\theta}^{(2)}(r)$ for the Li atom with $2+\alpha$ electrons, for various values of $\alpha$. The KS gap, $E_{g}^{\mathrm{KS}}$, is denoted on all panels for comparison.

LSDA, in their standard implementation do not reproduce the jump experienced by the KS potential when $N$ surpasses an integer value. But what about the Pauli potential in the LSDA?

Figure 2 presents results for the Pauli potential obtained from a spin-polarized LSDA calculation of Li with $2+\alpha$ electrons. The fraction of the electron, $\alpha$, has been added to the $\uparrow$-channel. In panel (a) one can see the $\uparrow$-Pauli potential, $v_{\theta, \uparrow}(r)$, for $\alpha=0,10^{-6}, 10^{-5}, 10^{-4}, 10^{-3}, 10^{-2}, 10^{-1}$, and 1. Clearly, the potential experiences a plateau, whose height approaches the LSDA KS gap for $\mathrm{Li}^{+}$of 1.9500 Hartree and whose width extends to infinity as $\alpha \rightarrow 0^{+}$. The rate of approaching the asymptotic height is rather fast: already for $\alpha=10^{-2}$ the plateau height of the potential is indistinguishable from its asymptotic value. The LSDA KS gap is quantitatively different from the exact gap value for $\mathrm{Li}^{+}$, but the mere phenomenon of a plateau in the Pauli potential is definitely present in the LSDA.

Panel (a) shows Pauli potentials numerically obtained in the two ways detailed in Sec. V: results for (i) are in color (see the legend), whereas results for (ii) are in white. Notably, results obtained by the two methods overlap everywhere. The $\downarrow$-channel of the Pauli potential shows no plateau and remains zero, as expected, and therefore it is not plotted here.

As to the ingredients of the LSDA Pauli potential for $\mathrm{Li}$, $v_{\theta, \uparrow}^{(1)}(r)$ and $v_{\theta, \uparrow}^{(2)}(r)$, we see the same situation as we observed in Sec. VIA: $v_{\theta, \uparrow}^{(2)}(r)$ is the term that creates the plateau, whereas $v_{\theta, \uparrow}^{(1)}(r)$ is merely a peak, which appears around the edge of the plateau, effectively shifting it to the right.

Finally, we consider the $\mathrm{Na}$ atom with $10+\alpha$ electrons. Unlike the Li case discussed above, the Pauli potential for 


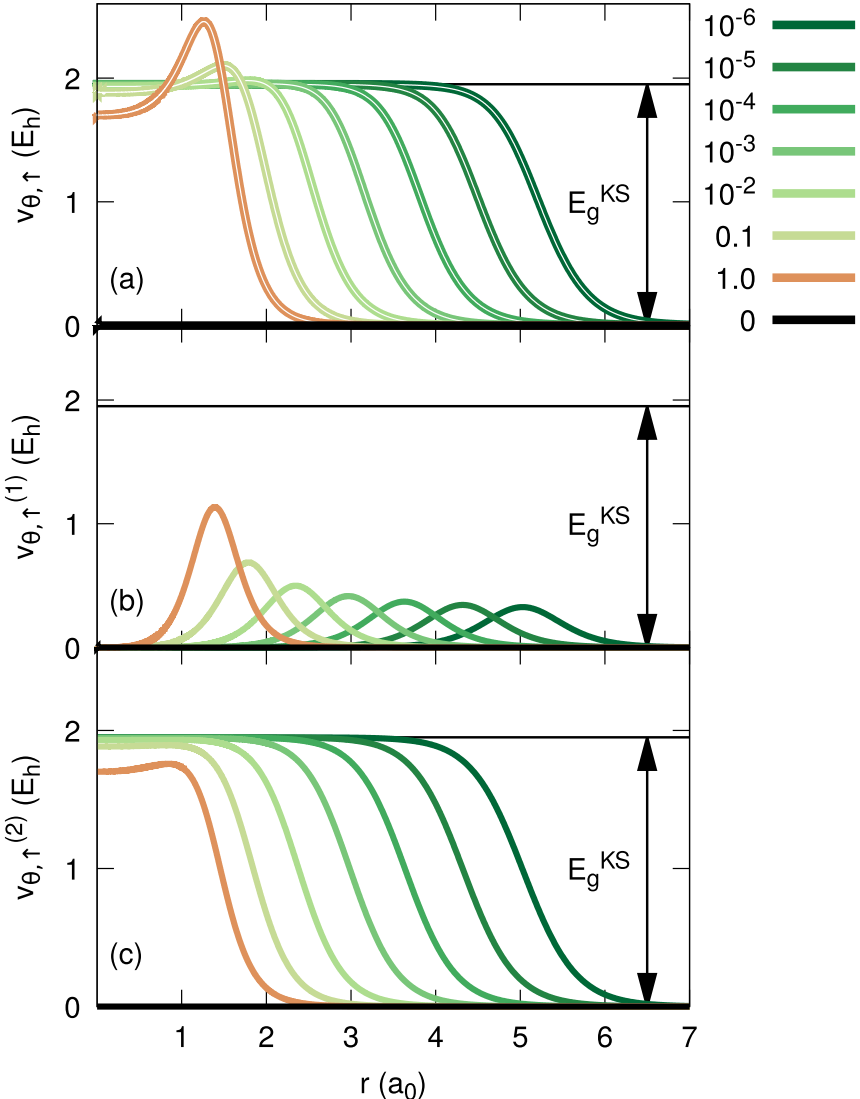

FIG. 2. (a) Pauli potential, $v_{\theta, \uparrow}(r)$, for the Li atom with $2+\alpha$ electrons, for various values of $\alpha$ (see the legend), obtained via Eq. (20) relying on an LSDA calculation. Pauli potentials obtained using Eq. (15) (white) completely overlap the above potentials. (b) The term $v_{\theta, \uparrow}^{(1)}(r)$ for the Li atom with $2+\alpha$ electrons, for various values of $\alpha$. (c) The term $v_{\theta, \uparrow}^{(2)}(r)$ for the Li atom with $2+\alpha$ electrons, for various values of $\alpha$. The LSDA KS gap of $\mathrm{Li}^{+}$is denoted in all panels for comparison.

the $\mathrm{Na}$ ion (i.e., for $\alpha=0$ ) is not zero, and therefore here we actually consider Pauli potential differences, $\Delta v_{\theta, \uparrow}(r)$, comparing the potential for a given $\alpha$ to its counterpart for $\alpha=0$. Unlike the Pauli potential itself, the potential difference can reach both positive and negative values. Hence, in Fig. 3(a) $\Delta v_{\theta, \uparrow}(r)$ first goes above the asymptotic value of $E_{g}^{\mathrm{KS}}$, then it goes below 0 , and finally it vanishes at infinity. Short of that, qualitatively the results for $\mathrm{Na}$ are very similar to those of Li: a pronounced plateau of height $E_{g}^{\mathrm{KS}}=1.0853$ Hartree in the Pauli potential, occurring due to $v_{\theta, \uparrow}^{(2)}(r)$ and extending to infinity as $\alpha \rightarrow 0^{+}$.

\section{CONCLUSIONS}

In this work, we explored the properties of the Pauli potential - a central quantity in orbital-free (OF) DFT and in the exact electron factorization (EEF) method. We expressed the exact Pauli potential in terms of the KS orbitals and energies for a spin-polarized system with a fractional number of electrons, using the ensemble approach in DFT. By this we generalized a previous exact result given in Ref. [17]. Furthermore, we found that as the number of electrons in

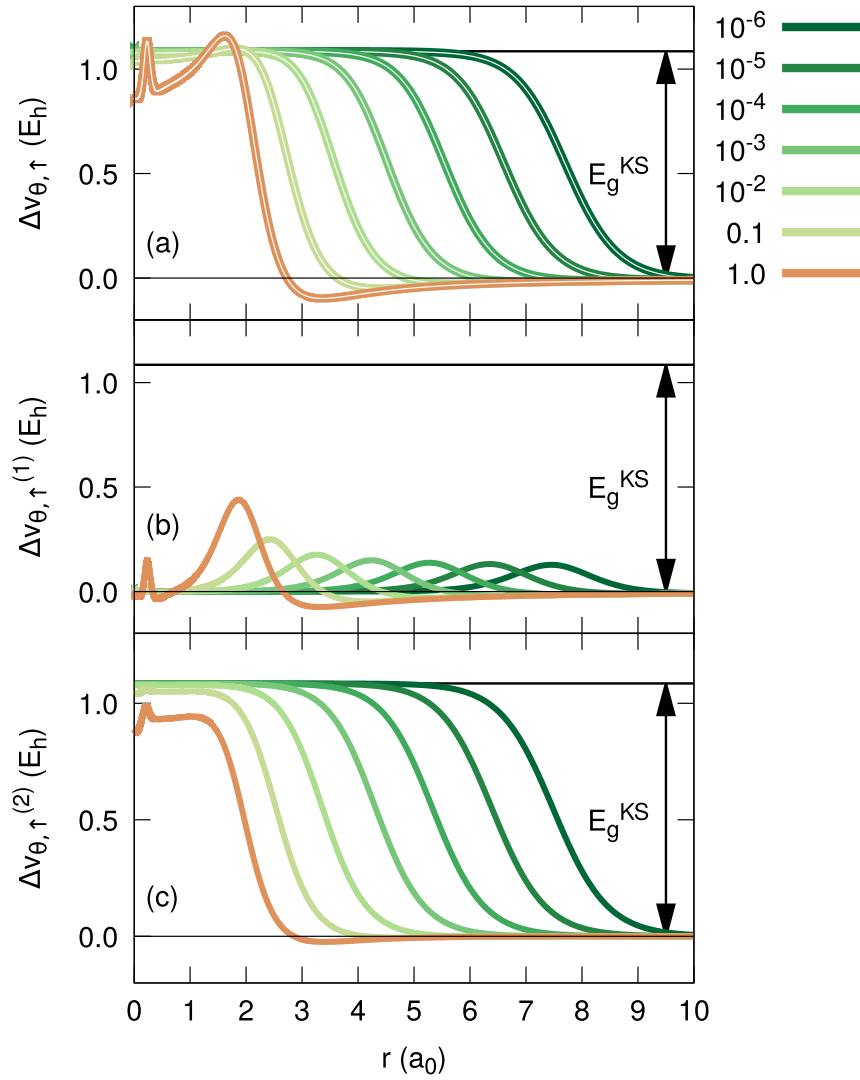

FIG. 3. (a) Pauli potential differences, $\Delta v_{\theta, \uparrow}(r)$, for the Na atom with $10+\alpha$ electrons, for various values of $\alpha$ (see the legend), obtained via Eq. (20) relying on an LSDA calculation. Pauli potential differences obtained using Eq. (15) (white) completely overlap the above potentials. (b) The term $\Delta v_{\theta \uparrow}^{(1)}(r)$ for the Na atom with $10+\alpha$ electrons, for various values of $\alpha$. (c) The term $\Delta v_{\theta, \uparrow}^{(2)}(r)$ for the $\mathrm{Na}$ atom with $10+\alpha$ electrons, for various values of $\alpha$. The LSDA KS gap of $\mathrm{Na}^{+}$is denoted in all panels for comparison.

the system surpasses an integer value, the Pauli potential experiences an abrupt jump; the magnitude of this jump equals the KS gap, $E_{g}^{\mathrm{KS}}$. These results have been illustrated for atomic systems, both for the exact case, which relies on full configuration-interaction calculations, and for the LSDA. Notably, we found that even such a simple xc approximation as the LSDA is capable of creating the abrupt jump in the Pauli potential, despite its inability to do the same for the KS potential. These findings can serve as benchmarks for future advanced approximations to the Pauli potential within OFDFT. Furthermore, since in KS-DFT the jump in the potential is closely related [50] to the step the potential produces in extended systems (e.g., stretched diatomic molecules), it is reasonable to expect a similar relationship also for the Pauli potential. This question, as well as the behavior of the Pauli potential in cases of dissociation and charge transfer, are possible directions for future research.

\section{ACKNOWLEDGMENT}

A. S. gratefully acknowledges financial support by an Ambizione grant of the Swiss National Science Foundation. 
[1] P. Hohenberg and W. Kohn, Phys. Rev. 136, B864 (1964).

[2] R. G. Parr and W. Yang, Density-Functional Theory of Atoms and Molecules (Oxford University Press, Oxford, 1989).

[3] R. M. Dreizler and E. K. U. Gross, Density Functional Theory (Springer-Verlag, Berlin, 1990).

[4] A Primer in Density Functional Theory, edited by C. Fiolhais, F. Nogueira, and M. A. L. Marques (Springer-Verlag, Berlin, Heidelberg, 2003)

[5] E. Engel and R. Dreizler, Density Functional Theory: An Advanced Course (Springer-Verlag, Berlin, Heidelberg, 2011).

[6] K. Burke, J. Chem. Phys. 136, 150901 (2012).

[7] R. M. Martin, Electronic Structure (Cambridge University Press, Cambridge, 2004).

[8] W. Koch and M. Holthausen, A Chemist's Guide to Density Functional Theory (Wiley-VCH, Weinheim, 2000).

[9] E. Kaxiras, Atomic and Electronic Structure of Solids (Cambridge University Press, Cambridge, 2003).

[10] C. Cramer, Essentials of Computational Chemistry: Theories and Models (Wiley, Chichester, England, 2004).

[11] D. Sholl and J. Steckel, Density Functional Theory: A Practical Introduction (Wiley-Interscience, Hoboken, NJ, 2011).

[12] W. Kohn and L. J. Sham, Phys. Rev. 140, A1133 (1965).

[13] For simplicity, a spin-unpolarized formalism is employed here.

[14] Hartree atomic units are used throughout.

[15] M. Levy, J. P. Perdew, and V. Sahni, Phys. Rev. A 30, 2745 (1984).

[16] N. H. March, Phys. Lett. A 113, 476 (1986).

[17] M. Levy and H. Ou-Yang, Phys. Rev. A 38, 625 (1988).

[18] Y. A. Wang and E. A. Carter, in Theoretical Methods in Condensed Phase Chemistry, edited by S. D. Schwartz (Springer, Berlin, 2002), pp. 117-184.

[19] V. Lignères and E. A. Carter, in Handbook of Materials Modeling, edited by S. Yip (Springer, The Netherlands, 2005), Chap. 1.8, pp. 137-148.

[20] J.-D. Chai, V. L. Lignères, G. Ho, E. A. Carter, and J. D. Weeks, Chem. Phys. Lett. 473, 263 (2009).

[21] V. V. Karasiev and S. B. Trickey, Comput. Phys. Commun. 183, 2519 (2012)

[22] H. Levämäki, Á. Nagy, K. Kokko, and L. Vitos, Phys. Rev. A 92, 062502 (2015).

[23] K. Finzel, Comput. Theor. Chem. 1144, 50 (2018).

[24] K. Finzel, J. Chem. Phys. 151, 024109 (2019).

[25] G. Hunter, Int. J. Quantum Chem. 29, 197 (1986).

[26] A. Schild and E. K. U. Gross, Phys. Rev. Lett. 118, 163202 (2017).

[27] A. Abedi, N. T. Maitra, and E. K. U. Gross, Phys. Rev. Lett. 105, 123002 (2010).

[28] A. Abedi, F. Agostini, Y. Suzuki, and E. K. U. Gross, Phys. Rev. Lett. 110, 263001 (2013).

[29] N. I. Gidopoulos and E. K. U. Gross, Trans. R. Soc. A 372, 20130059 (2014)

[30] E. Kraisler, Isr. J. Chem., doi:10.1002/ijch.201900103.

[31] V. V. Karasiev, R. S. Jones, S. B. Trickey, and F. E. Harris, Phys. Rev. B 80, 245120 (2009).

[32] K. Finzel, Theor. Chem. Acc. 134, 106 (2015).

[33] K. Finzel, Int. J. Quantum Chem. 115, 1629 (2015).

[34] K. Finzel, J. Chem. Phys. 144, 034108 (2016).
[35] K. Finzel, Int. J. Quantum Chem. 116, 1261 (2016).

[36] K. Finzel and M. Kohout, Theor. Chem. Acc. 137, 182 (2018).

[37] Q. Zhao and R. G. Parr, Phys. Rev. A 46, 2337 (1992).

[38] H. Levämäki, Á. Nagy, K. Kokko, and L. Vitos, Phys. Rev. A 90, 062515 (2014).

[39] Á. Nagy, Chem. Phys. Lett. 460, 343 (2008).

[40] Á. Nagy, J. Chem. Phys. 135, 044106 (2011).

[41] Á. Nagy and N. H. March, Int. J. Quantum Chem. 39, 615 (1991).

[42] J. P. Perdew and M. Levy, Phys. Rev. Lett. 51, 1884 (1983).

[43] L. J. Sham and M. Schlüter, Phys. Rev. Lett. 51, 1888 (1983).

[44] R. W. Godby, M. Schlüter, and L. J. Sham, Phys. Rev. B 36, 6497 (1987).

[45] R. W. Godby, M. Schlüter, and L. J. Sham, Phys. Rev. B 37, 10159 (1988)

[46] M. Allen and D. Tozer, Mol. Phys. 100, 433 (2002).

[47] W. Yang, A. J. Cohen, and P. Mori-Sánchez, J. Chem. Phys. 136, 204111 (2012).

[48] T. Gould and J. Toulouse, Phys. Rev. A 90, 050502(R) (2014).

[49] A. Görling, Phys. Rev. B 91, 245120 (2015).

[50] M. J. P. Hodgson, E. Kraisler, A. Schild, and E. K. U. Gross, J. Phys. Chem. Lett. 8, 5974 (2017).

[51] J. P. Perdew, R. G. Parr, M. Levy, and J. L. Balduz, Phys. Rev. Lett. 49, 1691 (1982).

[52] L. D. Landau and E. M. Lifshitz, Quantum Mechanics: NonRelativistic Theory, 3rd ed., Course of Theoretical Physics Vol. 3 (Pergamon Press, Oxford, 1991).

[53] E. H. Lieb, Int. J. Quantum Chem. 24, 243 (1983).

[54] A. J. Cohen, P. Mori-Sánchez, and W. Yang, Chem. Rev. 112, 289 (2012).

[55] The convexity conjecture means that the energies of all the (positive and negative) ions of the system, as well as the energy of the neutral system, form a mathematical series $E_{m}$ (with $m \in \mathbb{N}$ ), which is a convex, monotonously decreasing series. In other words, all ionization energies of the system are positive, $I_{m}>0$, and higher ionizations are always larger than the lower ones: $I_{m-1}>I_{m}$.

[56] R. van Leeuwen, Ph.D. thesis, Vrije Universiteit, Amsterdam, The Netherlands (1994).

[57] R. van Leeuwen, Adv. Quantum Chem. 43, 25 (2003).

[58] E. Kraisler and L. Kronik, Phys. Rev. Lett. 110, 126403 (2013).

[59] E. Kraisler and L. Kronik, J. Chem. Phys. 140, 18A540 (2014).

[60] These ground states are assumed to be nondegenerate.

[61] J. F. Janak, Phys. Rev. B 18, 7165 (1978).

[62] In this section, for the sake of brevity, the KS eigenvalues and orbitals are not accompanied anymore by the superscript $(\alpha)$. Still, we keep in mind their implicit dependence on the number of electrons in the system.

[63] H.-J. Werner, P. J. Knowles, G. Knizia, F. R. Manby, M. Schütz et al., MOLPRO, version 2015.1, a package of ab initio programs (2015), available on www.molpro.net.

[64] E. V. R. de Castro and F. E. Jorge, J. Chem. Phys. 108, 5225 (1998).

[65] A. P. Gaiduk, I. G. Ryabinkin, and V. N. Staroverov, J. Chem. Theory Comput. 9, 3959 (2013).

[66] G. Hermann, V. Pohl, J. C. Tremblay, B. Paulus, H.-C. Hege, and A. Schild, J. Comput. Chem. 37, 1511 (2016). 
[67] M. J. P. Hodgson, J. D. Ramsden, J. B. J. Chapman, P. Lillystone, and R. W. Godby, Phys. Rev. B 88, 241102(R) (2013).

[68] E. Kraisler, G. Makov, N. Argaman, and I. Kelson, Phys. Rev. A 80, 032115 (2009).
[69] E. Kraisler, G. Makov, and I. Kelson, Phys. Rev. A 82, 042516 (2010).

[70] J. P. Perdew and Y. Wang, Phys. Rev. B 45, 13244 (1992).

[71] $\mathrm{Li}^{+}$has only one doubly occupied $\mathrm{KS}$ orbital, $\varphi_{1}(\mathbf{r})$. Hence, $q_{1}(\mathbf{r})=$ const, $\varepsilon_{1}=\varepsilon_{\mathrm{ho}}$, and therefore $v_{\theta}(r)=0$. 